\title{
ADMINISTRACIÓN DE LA INFRAESTRUCTURA ESTRATÉGICA EN EL SECTOR DEFENSA DE COLOMBIA. RELACIÓN CON LA ESTRATEGIA ADOPTADA POR ESPAÑA*
}

\section{ADMINISTRATION OF STRATEGIC INFRASTRUCTURE IN THE DEFENSE SECTOR OF COLOMBIA. RELATIONSHIP WITH THE STRATEGY ADOPTED BY SPAIN}

Recibido: 10 de septiembre de 2020

Evaluado: 15 de octubre de 2020

Aprobado: 11 de diciembre de 2020

Sandra Marcela Avila Bernal ${ }^{*}$

Universidad Externado de Colombia

Orcid: https://orcid.org/0000-0001-8242-6686

Cómo citar este artículo: Avila Bernal, S.M. (2021). ADMINISTRACIÓN DE LA INFRAESTRUCTURA ESTRATÉGICA EN EL SECTOR DEFENSA DE COLOMBIA. RELACIÓN CON LA ESTRATEGIA ADOPTADA POR ESPAÑA. Revista Estrategia Organizacional, 10(1), https://doi.org/10.22490/25392786.4552

* $\quad$ Artículo de investigación.

** $\quad$ Magister en Gestión Social Empresarial. 


\section{RESUMEN}

Introducción: Este artículo realiza un análisis a lo conexo con la protección de la Infraestructura Estratégica - Crítica desde el Sector Defensa. Entiende el fenómeno que concibe su protección, e identifica las lecciones aprendidas que permiten, incluso, ubicar el punto de partida en el que el sector a través de la Fuerza Pública puede encontrarse, de acuerdo con parámetros comparativos del caso España. Metodología: se realizó una entrevista a personal experto en temas de seguridad y defensa, así como en temas de seguridad pública y de infraestructura, con preguntas abiertas y cerradas a quienes se encontraban en un Nivel Directivo, Medio y Operacional, con el fin de poder recoger tanto su conocimiento, experticia y su visión de la temática propuesta, y así, obtener resultados objetivos, críticos y confiables. También, se estructuró una lista de chequeo con el fin de lograr realizar una comparación de las principales características que permitan verificar el grado de desarrollo del Programa de Protección de Infraestructura Crítica con que cuenta actualmente España, frente a lo encontrado en el Sector Defensa y con el fin de acotar la fase de confirmación y validación de la investigación que se realizó a través del Grupo de Expertos consultados, mediante una retroalimentación de los resultados obtenidos. Resultados: La presente investigación, además de tener un matiz diferenciador de las temáticas normalmente abordadas, permite resaltar que las ciencias sociales son fundamentales para la investigación de Infraestructura Estratégica - Crítica, de manera transdisciplinaria de varias formas. En este sentido, se intentó considerar a lo largo de esta investigación "la importancia de la seguridad y defensa como agentes del desarrollo económico estable y sostenible" (Medeiros \& Pereyra Bordón, s. f., p.115). Permitiendo suministrar, descripciones que serán esenciales para generar juicios de transferibilidad' al contexto del Sector Defensa sobre la protección y administración de la Infraestructura Estratégica - Crítica, situación concreta con características singulares.

Palabras clave: Infraestructura, estratégia, protección, catalogo, crítica, administración.

\section{ABSTRACT}

Introduction: This article performs an analysis related to the protection of the Strategic Infrastructure - Critical from the Defense Sector. It understands the phenomenon that its protection conceives, and identifies the lessons learned that allow, even, to locate the starting point in which the sector

1 Según Wikifinanzas: El término de transferibilidad indica la velocidad con la que los competidores pueden imitar los procesos en que se fundamenta la ventaja competitiva de una empresa. 
through the Public Force can find itself, according to comparative parameters of the case of Spain. Methodology: an interview was conducted with expert personnel in security and defense issues, as well as in public security and infrastructure issues, with open and closed questions to those who were at a Management, Medium and Operational Level, in order to be able to collect both their knowledge, expertise and their vision of the proposed topic, and thus, obtain objective, critical and reliable results. Also, a checklist was structured in order to achieve a comparison of the main characteristics that allow verifying the degree of development of the Critical Infrastructure Protection Program that Spain currently has, compared to what was found in the Defense Sector and with in order to limit the confirmation and validation phase of the research that was carried out through the Group of Experts consulted, through feedback on the results obtained. Results: The present research, in addition to having a differentiating nuance from the topics normally addressed, makes it possible to highlight that the social sciences are fundamental for the research of Strategic-Critical Infrastructure, in a transdisciplinary way in several ways. In this sense, an attempt was made to consider throughout this research "the importance of security and defense as agents of stable and sustainable economic development" (Medeiros \& Pereyra Bordón, s. F., P.115). Allowing to supply, descriptions that will be essential to generate transferability judgments to the context of the Defense Sector on the protection and administration of the Strategic - Critical Infrastructure, a concrete situation with unique characteristics.

Keywords: Infrastructure, strategic, protection, catalog, criticism, categorization.

\section{INTRODUCCIÓN}

La infraestructura Estratégica - Crítica tiene una incidencia significativa en lo relacionado con los procesos económicos de un país, sumado a la percepción que el Estado y en general la sociedad, deba gestionar medidas de seguridad que sustente la confianza de usuarios de los servicios que cubran las funciones básicas de un territorio, a través de la utilización de algún tipo de infraestructura Estratégica - Crítica, pues implica una movilización que direccione esfuerzos significativos para lograr el acceso y mantenimiento de este tipo de infraestructura. Es por esto que a nivel mundial, su protección y conservación se convierten en líneas de acción estatales, que vinculan de manera preponderante varios actores relacionados con los procesos de prestación 
de servicios y protección. En este sentido, esta investigación si bien no logra teorizar, si dará más elementos a considerar para abordar esta temática, desde la investigación social y con el fin de poder establecer la 'Protección de la Infraestructura estratégica para Colombia en el contexto del Sector Defensa en relación con la Estrategia adoptada por España', por la incidencia que tiene esta infraestructura en los servicios esenciales prestados a la sociedad, además de posibles perturbaciones y alteraciones graves en materia de seguridad.

Como objetivo general la presente investigación buscó establecer aspectos a considerar para la protección de la 'Infraestructura Estratégica para Colombia en el contexto del Sector Defensa en relación con la Estrategia adoptada por España' y del cual se desplegaron tres (3) objetivos específicos: 1. Analizar cómo la Infraestructura Estratégica tiene relación con las necesidades humanas, su satisfacción y temas de estrategia, defensa y seguridad. 2. Examinar la Estrategia de Protección de la Infraestructura Crítica adoptada por España como un referente para Colombia en el contexto del Sector Defensa. 3. Proponer líneas de acción que contribuyan al Sector Defensa a fortalecer la Protección de los Activos Estratégicos de la Nación.

También se desarrolló una Metodología basada en el trabajo de Sanabria Rangel (Sanabria Rangel, 2016), con un estilo o perspectiva interpretativa que permitió abordar el problema social, y que ha sido dirigida a generar conocimiento con ciertas características partiendo de la experiencia y aportando a la solución de un problema específico identificado. En cuanto al enfoque, fue de tipo cualitativo, teniendo en cuenta que se buscó dar respuesta al problema de investigación y a los objetivos de esta, asimismo, se desarrolló dentro un contexto concreto por lo tanto su muestreo fue intencional y no representa un tema o una población de forma estadística únicamente, puesto que la muestra se obtuvo mediante la colaboración de diferentes actores que por su experiencia aportaron datos relevantes al objeto de estudio (Colás Bravo \& De Pablos Pons, 2012, p. 78).

Sobre la Temporalidad, esta fue transversal, pues los datos se recogieron sobre un grupo en un solo momento temporal (Buendía Eisman et al., 1998, p.153). El procedimiento utilizado fue el sintético en este entendido, vale la pena entonces complementar lo indicado por Sanabria con lo argumentado por Maya (2014) “Es el que analiza y sintetiza la información recopilada, lo que permite ir estructurando las ideas" (Maya, 2014, p.13). El modo fue deductivo, de tipo descriptivo que obedece a "una interpretación subjetiva, pero no es arbitraria" (Abreu, 2014, p.199), con una 
aproximación empírica basada en la experiencia. Su diseño no fue experimental, sino basada en el estudio de caso, debido a que existía una situación específica que se abordó, la cual contó con características particulares que a su vez integró diferentes actores y permitió inferir la organización a la que se busca intervenir, así como los sectores sociales y a la Fuerza Pública a la cual impactará, estos últimos, son quienes tienen la responsabilidad directa de la protección efectiva de los Activos Estratégicos de la Nación. La técnica utilizada fue la observación no participante - entrevista, una lista de chequeo con el fin de acotar la confirmación y validación de la investigación a través del Grupo de Expertos consultados y en virtud de abordar la fase planteada por el autor Hernández Sampieri (2004) cuando hace referencia a que:

Un tercer elemento para asegurar la confiabilidad y validez de los resultados (que no siempre es posible aplicar), consiste en obtener retroalimentación directa de los sujetos de la investigación (al menos una muestra de ellos), lo cual significa pedirles que confirmen o refuten nuestras interpretaciones y ver si capturamos los significados que ellos trataban de transmitir, así como si nuestras descripciones fueron lo suficientemente completas y profundas (Hernández Sampieri et al., 2004, p.482).

Así mismo, los medios de análisis de información fueron a través de la organización de información y la utilización de matrices de Excel y el software Nvivo12.

\section{METODOLOGÍA}

Para dar cumplimiento a los objetivos trazados ya descritos, se elaboró este apartado sustentado en los siguientes pilares: 1. Seguridad y Defensa elementos necesarios para sustentar los servicios esenciales de la sociedad: con el fin de entender el concepto ligado a esta protección desde la Seguridad y Defensa, pero también desde la satisfacción de las necesidades humanas. Para fortalecer esta idea fue útil compartir una teoría psicológica propuesta por Abraham Maslow y citada por los autores Medeiros y Pereyra (2015), la cual fue desarrollada a través de una pirámide de cinco niveles denominada 'de las necesidades'.

Su análisis comienza en las llamadas necesidades básicas, propias de las personas, parte que se encuentra en la base de la pirámide con el concepto de que estas son las que dan sustento al resto de las otras necesidades. La segunda capa de esta pirámide se vincula a la necesidad de 
seguridad y protección, esta incluye la seguridad física y mental, la seguridad de contar con los recursos para vivir, educación sanidad, asimismo, este estrato incluye la necesidad de proteger nuestras pertenencias. La tercera capa está definida por las necesidades sociales, como la de pertenencia a un grupo, necesidad de relacionarse, y de ser aceptado. La cuarta capa hacia arriba es llamada necesidad de autoestima, como tener atención, respeto, reconocimiento por los demás y éxito, entre otros. El tope de la pirámide y última capa es la necesidad de crecimiento, de darle sentido a la vida, a la propia existencia, de sentirse realizado (Medeiros \& Pereyra Bordón, s. f., p. 115).

Una vez abordado el concepto de seguridad desde la perspectiva de necesidad bajo la teoría de Abraham Maslow y a través del planteamiento realizado en la investigación "Colaboración Sector Defensa Pública en confrontación a las vulnerabilidades de seguridad hemisféricas" se pudo concluir que las necesidades básicas humanas, están relacionadas con aspectos de defensa y seguridad y que estos en su conjunto, son una parte esencial de la productividad de las relaciones económicas. Por ende, es posible establecer que la Protección de la Infraestructura Estratégica - Crítica hace parte de este conjunto, fenómeno que fue detallado a lo largo de la presente investigación y se convierte en gran medida en un medio satisfactor para cubrir este tipo de necesidades.

Entendido lo anterior, a continuación, se abordarán algunas reflexiones sobre el enfoque planteado por Manfred Max - Neef sobre "El desarrollo a escala humana una opción para el futuro", con el fin de identificar aspectos importantes que den valor a la relevancia que tiene la Defensa y Seguridad en la Protección de la Infraestructura Estratégica - Crítica y esta a su vez, en la incidencia que pueda tener en la satisfacción de las necesidades humanas fundamentales planteadas por este autor.

De este apartado, vale la pena resaltar que el desarrollo se refiere a las personas y no a los objetos, entonces: "El mejor proceso de desarrollo será aquel que permita elevar más la calidad de vida de las personas". Ahora, la siguiente pregunta que se plantea es: "¿Qué determina la calidad de vida de las personas?". Al respecto Max-Neff (2010) manifiesta que: "La calidad de vida dependerá de las posibilidades que tengan las personas de satisfacer adecuadamente sus necesidades humanas fundamentales" (Manfred et al., 2010, p.25). 
En conclusión "El Desarrollo a Escala Humana no excluye metas convencionales como crecimiento económico para que todas las personas puedan tener un acceso digno a bienes y servicios"(Manfred et al., 2010, p.51) y así, afırmar que la infraestructura que sustenta el funcionamiento de los servicios esenciales de los sectores estratégicos que en algún momento son clasificados como críticos, son fundamentales para el desarrollo de la humanidad, teniendo como premisa lo ya planteado, la defensa y seguridad de la Infraestructura Estratégica - Crítica, que juegan un papel fundamental en la protección de las infraestructuras que las conforman, en el sostenimiento fiscal de la nación y en los recursos que proporcionan la inversión social en el territorio, entre otros aspectos.

Vinculado a los conceptos abordados y a la temática que se pretendió desplegar a lo largo de esta investigación, se consideraron como importantes los aportes investigativos contenidos en la Energy Research \& Social Science (ERSS), una revista internacional revisada por pares académicos que publica investigaciones originales y artículos de revisión encaminados a examinar la relación entre los sistemas de energía y la sociedad. (Aims \& scope - Energy Research \& Social Science I ScienceDirect.com, s. f.). Su contenido permitió un acercamiento de tipo investigativo y académico que vinculó significativamente, argumentos que pocos investigadores refieren en materia de Infraestructura Estratégica - Crítica y su incidencia en los procesos sociales evidenciando la importancia de su preservación o protección.

Para tal efecto, la investigación 'Transforming power: Social science and the politics of energy choices' o 'Transformando el poder: La ciencia social y la política de las opciones energéticas' Es un documento que aborda las implicaciones claves, tanto para la ciencia social como para la sociedad" (Stirling, 2014, p.83).

Sterling (2014) indica que la comunidad de investigadores que se dedican específicamente al estudio científico social de los sistemas energéticos es relativamente pequeña:

A pesar de que desde hace mucho tiempo se ha reconocido el papel de la investigación social en el estudio de la energía y no hay duda de su importancia. Pero lo contrario también es cierto. De los muchos "sectores" funcionales convenientemente segregados, pero íntimamente interconectados, de la vida socioeconómica (como el agua, la alimentación 
y la vivienda), también existe un sentido en el que ninguno es más importante para el desarrollo general de las ciencias sociales en general, o incluso para la propia sociedad, que la "energía" (Stirling, 2014, p.84).

En ese mismo contexto, este tipo de infraestructura y la sociedad libran luchas para lograr transformaciones culturales, infraestructurales, políticas y económicas profundas, evidenciando desafíos mundiales en una escala significativamente mayor a abordar por parte de los países y los gobiernos encargados de asumirlos.

Generalidades de la Protección de la Infraestructura Estratégica - Crítica: Como ya se ha mencionado la Infraestructura Estratégica sustenta el funcionamiento de los servicios esenciales para el mantenimiento de las funciones básicas de la sociedad. La Infraestructura Crítica, entonces, son activos de Infraestructura Estratégica que han sido clasificados como críticos bajo ciertas características de categorización. De este modo, a continuación, se abordó la investigación 'Critical infrastructure protection: Requirements and challenges for the $21 s t$ century' o 'Protección de infraestructura crítica: requisitos y desafíos para el siglo XXI', publicada por la Revista Internacional de Protección de Infraestructura Crítica, donde las autoras Alcaraz y Zeadally (2015), puntualizan que:

Las infraestructuras críticas juegan un papel vital en el apoyo a la sociedad moderna. La fiabilidad, el rendimiento, el funcionamiento continuo, la seguridad, el mantenimiento y la protección de las infraestructuras críticas son prioridades nacionales para los países de todo el mundo (Alcaraz \& Zeadally, 2015, p.53).

Así mismo las autoras citan lo indicado por U.S. General Accounting Office, Critical Infrastructure Protection, (2011), donde señalan que:

Una infraestructura crítica comprende los sistemas y activos, ya sean físicos o virtuales, que son tan esenciales para una nación que cualquier interrupción de sus servicios podría tener un grave impacto en la seguridad nacional, el bienestar económico, la salud o la seguridad pública, o cualquier combinación de estos (Alcaraz \& Zeadally, 2015, p.53). 
Las autoras permiten evidenciar que existen conexiones entre sectores críticos de infraestructura, los cuales producen relaciones especiales de interdependencia y por ende, podrían desencadenar efectos en cascada en múltiples infraestructuras críticas, que se podrían materializar cuando una infraestructura crítica sufre algún tipo de alteración, daño o destrucción. Por lo anterior, lo relacionado con la protección de la infraestructura crítica se debe considerar como una actividad intersectorial y no es recomendable limitarla a sectores específicos aislados de la articulación gubernamental con otros, sino por el contrario, se debe asumir de manera coordinada bajo una perspectiva holística (Alcaraz \& Zeadally, 2015, p.54).

Una conclusión de este segmento de acuerdo con lo dicho por las autoras es que "Ios aspectos críticos para la seguridad deben considerarse para controlar los efectos en cascada". Además, que "La propiedad de la seguridad crítica implica evitar o mitigar la propagación de efectos entre infraestructuras críticas, lo que podría provocar daños físicos y fisiológicos, lesiones y muertes" (Alcaraz \& Zeadally, 2015, p.57) así mismo, que la diversidad de las infraestructuras críticas, requieren el diseño y despliegue de numerosas medidas de protección. Los esfuerzos de protección deberían centrarse en los mecanismos de seguridad tradicionales para detectar y responder a las amenazas, así como en sistemas inteligentes que puedan identificar de manera proactiva vulnerabilidades y fallas que los atacantes puedan explotar.

Iniciativas existentes en Protección de Infraestructuras Críticas en EE. UU, Unión Europea España: con el fin de lograr obtener un contexto general, se estudió sobre las generalidades de las estrategias planteadas por países como EE.UU. y los de la Unión Europea, por ser los pioneros en este campo y de donde se desliga algunos apartados de los temas abordados por España, es importante mencionar que de la literatura encontrada en este campo, este país, es uno de los pocos, donde es posible acceder a apartes de la información, debido a que en ocasiones esta es manejada bajo normas de reserva de la información.

Es así, como parte de la revisión del estado del arte de lo relacionado con la Protección de la Infraestructura Crítica (PIC), obliga a involucrar un referente internacional en el intercambio de conocimiento, experiencias y la dinamización de los sectores involucrados en este ámbito, como lo es el Centro de Ciberseguridad Industrial (CCl) organización independiente sin ánimo de lucro de España, que ha venido desarrollando actividades de análisis, estudios e intercambio 
de información sobre el conjunto de prácticas, procesos y tecnologías, en lo concerniente al ciberespacio e infraestructuras industriales o también conocidas como infraestructuras físicas de las organizaciones. Por lo anterior, la presente investigación apeló a la utilización de la recopilación del estado del arte, planteado por $\mathrm{CCI}$ contenido en la primera edición de su libro 'La Protección de Infraestructuras Críticas y la Ciberseguridad Industrial', donde reúne un estudio responsable sobre lo existente en esta materia. En primer lugar, se acota que para el $\mathrm{CCl}$ el término Infraestructura Crítica:

Es empleado por los Estados para definir instalaciones y sistemas sobre los que recaen servicios esenciales cuyo funcionamiento no permite soluciones alternativas. Las infraestructuras críticas existentes en un estado se agrupan dentro de sectores estratégicos: aquellos que son esenciales para la seguridad nacional o para el conjunto de la economía de un país (Centro de Ciberseguridad Industrial, 2013, p. 8).

El análisis de los aspectos teóricos y conceptuales permitió que la revisión se centrara en las diferentes estrategias de protección a la infraestructura crítica y recursos clave, presentando las experiencias en países considerados como de referencia internacional sobre el tema. En tal sentido, para poder entrar en materia e identificar las características de la Estrategia de Protección a la Infraestructura Crítica de España, fue necesario abordar como objeto de estudio la 'Estrategia de Seguridad Nacional 2017', permitiendo inferir que los modelos implementados e identificados hasta el momento por cada país, cuentan con marcos de actuación ligeramente diferentes, pero que buscan en general establecer lineamientos para fortalecer la seguridad de las infraestructuras y gestionar los riesgos asociadas a ellas. Se podría afirmar, que, a pesar de las diferencias puntuales o características enunciadas, también cuentan con muchas características comunes entre estos modelos, así como un extenso reconocimiento de la dependencia asociada a la infraestructura en escenarios como la seguridad nacional y el bienestar económico de los países, que requieren un liderazgo desde los más altos niveles. En este punto, de acuerdo con lo investigado por Galindo (2014):

Los sistemas: español, francés y japonés son más sencillos que el estadounidense, su estructura resulta más simple y, a priori, la información pasa por canales más fluidos, acortando los tiempos de reacción ante las amenazas (Galindo Sierra, 2016, p.34) 
De acuerdo con la información existente en los diferentes países y en especial en España, el bienestar social, la prosperidad de su economía y lo relacionado a la seguridad ciudadana y defensa nacional territorial de los países, está bastante influenciada por la Infraestructura Estratégica, catalogada como crítica. Es por ello, por lo que propender con su protección está directamente relacionada con retos estatales que ameritan medidas dinámicas, de acuerdo con su complejidad y al surgimiento de diferentes focos que puedan amenazar o convertirse en riesgos potenciales para los mismos.

Finalmente, en la investigación realizada sobre 'Protección de Infraestructuras Críticas: Un Análisis de Derecho Comparado' el autor Galindo (2016) manifiesta que, en definitiva, todos los implicados en la Protección de la Infraestructura Crítica, deben colaborar a la hora de mejorar los sistemas de protección. También indica que es necesaria la participación de la comunidad científica especializada en materia de seguridad, ya que son muchas las líneas de investigación posibles en relación a las mismas (aun teniendo la dificultad que supone el hecho de que gran parte de la información esté clasificada como secreta: tanto estudios cualitativos como cuantitativos sobre la efectividad de los planes que se llevan a cabo, la eficacia en la reacción en caso de amenaza, la proporcionalidad de las actuaciones realizadas, etc.). Estas investigaciones podrían contribuir a la mejora de los sistemas de protección de Infraestructura Crítica en los países (Galindo Sierra, 2016, p.36).

\section{INSTRUMENTOS}

Con base en las técnicas mencionadas anteriormente, los principales instrumentos empleados en la presente investigación fueron los siguientes: se realizó una entrevista a personal experto en temas de seguridad y defensa, así como en temas de seguridad pública y de infraestructura, con preguntas abiertas y cerradas a quienes se encontraban en un Nivel Directivo, Medio y Operacional, con el fin de poder recoger tanto su conocimiento, experticia y su visión de la temática propuesta, y así, obtener resultados objetivos, críticos y confiables. También, se estructuró una lista de chequeo con el fin de lograr realizar una comparación de las principales características que permitan verificar el grado de desarrollo del Programa de Protección de Infraestructura Crítica con que cuenta actualmente España, frente a lo encontrado en el Sector Defensa y con el fin de acotar la fase de confirmación y validación de la investigación que se realizó a través del Grupo de Expertos consultados, mediante una retroalimentación de los resultados obtenidos. 
Adicional al Programa Excel, que permitió visualizar apartes del análisis de las respuestas obtenidas, fue utilizado el Software NVivo 12. Dicho software, permitió realizar la organización de la información a través de sus herramientas de clasificación, conjuntos, nodos, casos y codificación entre otros. Con el fin de emprender procesos como el conteo de palabras, la búsqueda específica de ellas, así como la diagramación de nubes de palabras y ramificación de estas, entre otras bondades con las cuales cuenta el programa, en busca de preparar la información para su análisis.

\section{RESULTADOS}

Una vez realizada la consulta general en el software NVivo 12, se ejecutó el mismo procedimiento descrito en el anterior apartado, con cada una de las subcategorías y las preguntas relacionadas con ellas, con el fin de obtener árboles de palabras de acuerdo a los parámetros determinados para cada caso y a los diferentes nodos o codificaciones, así como los porcentajes de cobertura de cada palabra en cada uno de los casos registrados, lo que permitió identificar una serie de conexiones que junto a una Matriz General de Entrevistas construida en Excel, donde se registró todas las respuestas obtenidas por parte de los entrevistados, así como una Matriz General de Análisis, de cada una de las particularidades identificadas en las respuestas, se logra analizar la información para la obtención de los resultados descritos a continuación, contribuyendo al logro del objetivo general de la presente investigación.

En ese sentido, el desarrollo de esta investigación permitió identificar que el Sector Defensa requiere fortalecer la capacidad de enfoques que economicen esfuerzos institucionales y que generen compromisos con los agentes involucrados en la protección de la Infraestructura Estratégica - Crítica, consolidando acciones conjuntas y articuladas que contribuyan en el despliegue de labores en materia de seguridad, que favorezcan de manera directa el acceso y mantenimiento de infraestructuras que proporcionan servicios esenciales para los ciudadanos e involucran las diferentes actividades económicas y productivas del país.

Resultados de las necesidades relacionadas con la seguridad: Al realizar el análisis de esta Subcategoría, se logra establecer que efectivamente existe una relevancia importante respecto a las necesidades relacionadas con la seguridad, encontrando que los entrevistados coinciden con la afırmación que: “No existe la posibilidad de desarrollo si no está bajo el paraguas de la seguridad" (Medeiros \& Pereyra Bordón, s. f., p. 116 -117), (Forigua Rojas, 2020,p.1) (ND2, 2020,p.1) (NM1, 
2020,p.1) (ND3, 2020.p.1) (NO1, 2020,p.1). Así mismo, indican que el “Concepto de seguridad es una de las variables para tener en cuenta siempre, porque incide en cualquier actividad económica, ya que garantiza la sostenibilidad y tranquilidad en el desarrollo económico de cualquier sociedad al interior de un país" (ND2, 2020, p.1).

En relación con la teoría planteada, se fortalece la afirmación que "la seguridad hace parte del logro en una sociedad para crecer y desarrollarse" (Medeiros \& Pereyra Bordón, s. f., p. 116 -117), por lo anterior, se infiere de la importancia que esta representa en el componente humano respecto a la pirámide de necesidades expuesta, sin olvidar efectivamente, que este tipo de conceptos están mediados por las condiciones de percepción de cada persona.

En esta categoría también cabe destacar lo manifestado por el Coordinador del Grupo de Infraestructura Crítica consultado: "se encuentra una relación entre diferentes contextos y las estrategias de defensa nacional, en la medida que se logra identificar que la infraestructura estratégica presta servicios esenciales a la sociedad, así mismo hacen parte de la protección y la sostenibilidad de diferentes sectores económicos a nivel nacional" (ND2, 2020, p.2).

En efecto, como lo afirmó los autores Correa \& Yusta, los diferentes contextos se encuentran directamente relacionados con estrategias de defensa nacional, y es allí, donde uno de los expertos consultados, identifica que incluso están relacionados con "el bienestar de la nación y por ende de sus ciudadanos, ya que [...] hablamos de la integridad de la soberanía" (ND3, 2020, p.2).

El experto Consultor en Temas de Seguridad Pública y de Infraestructura también indica que:

"permite un óptimo desarrollo de los diferentes campos del poder, resultando en una mejor prestación de servicio y un mejor disfrute de estos, además, de una constante mejora de la oferta. Al final sería la sanidad fiscal, el empoderamiento del estado, el sentido de unidad o patriotismo y la minimización de las variables negativas que ataquen al estado" (ND3, 2020, p.2). 
De acuerdo con lo anterior, se ratifica la interpretación realizada en el aparte teórico referente y se puede concluir que las necesidades básicas humanas están relacionadas con aspectos de defensa y seguridad, y que estos en su conjunto, son una parte esencial de la productividad de las relaciones económicas, estableciendo así, que la Protección de la Infraestructura Estratégica Crítica hace parte de este conjunto.

Resultado apartado: Infraestructura Estratégica como medio por el cual el sujeto potencia los satisfactores para vivir sus necesidades: En la presente Subcategoría se buscó desarrollar diferentes apartes teóricos investigados. Inicialmente se planteó la siguiente afirmación a los expertos entrevistados: "La calidad de vida dependerá de las posibilidades que tengan las personas de satisfacer adecuadamente sus necesidades humanas fundamentales" (Manfred et al., 2010, p.25), con el objeto de interpretar la incidencia que pueda tener en la satisfacción de las necesidades humanas fundamentales planteadas por el Autor Max-Neff, con la Defensa y Seguridad en lo referente a la Protección de la Infraestructura Estratégica - Crítica.

Ahora bien, los expertos lograron identificar aspectos importantes en esta incidencia, como lo planteado por el Dr. Forigua, cuando indica que este tema "ha estado presente en temas de desarrollo y también cuando se miran como enfoques de seguridad humana o de seguridad multidimensional" (Forigua Rojas, 2020, p.2) haciendo énfasis en que "todo esquema debería partir de las necesidades humanas básicas satisfechas o por satisfacer" (Forigua Rojas, 2020, p.2). Lo que "elevaría la calidad de vida de las personas" (Forigua Rojas, 2020, p.2) porque como lo explica la Asesora de Seguridad Pública y de Infraestructura "Ios seres humanos y especialmente nuestros núcleos familiares estamos dirigidos a satisfacer nuestras necesidades básicas insatisfechas" (NM1, 2020, p.1)

Del mismo modo, el Director de Operaciones consultado, indica que "la satisfacción de las necesidades es un concepto algo subjetivo. Normalmente se evalúa desde el punto de vista de cada ser humano" (NO1, 2020, p.2) y en "términos de calidad de vida, evaluar las necesidades fundamentales podrían dar una idea sobre su cubrimiento o satisfacción y esta dependerá del acceso que una comunidad tenga a los servicios que suministre la infraestructura estratégica". (NO1, 2020, p.2). 
De igual forma, el Coordinador del Grupo de Infraestructura Crítica manifiesta que "al garantizar las necesidades fundamentales, las personas logran desarrollar sus actividades diarias sin limitaciones y fomenta el desarrollo de la sociedad" (ND2, 2020, p.2). Además, aquí se podría afirmar que la Infraestructura Estratégica como medio por el cual el sujeto potencia los satisfactores para vivir sus necesidades, hace parte de las "diversas maneras para ir desapareciendo el índice de pobreza multidimensional, que es un valor de referencia del bienestar a nivel internacional, abarcando la extensión del estado" (ND3, 2020, p.3.) como afirma el Consultor en Temas de Seguridad Pública y de Infraestructura.

Al relacionar la calidad de vida con lo concerniente a los satisfactores de las necesidades propuestos teóricamente por Max-Neff y para la presente investigación, se infiere como ya se ha venido afirmando, que la Infraestructura Estratégica sustenta el funcionamiento de los servicios esenciales para la vida de una comunidad, como una afirmación paralela a este planteamiento, así lo manifiestan los expertos consultados al indicar que, "suministra en gran parte servicios esenciales los cuales son vitales para el desarrollo de la sociedad y apoya el desarrollo económico de un país" (ND2, 2020, p.2) y adicionalmente, "es donde se realizan y se desarrollan todas las actividades productivas del país" (NM1, 2020, p.1), permitiendo sustentar "no solo los servicios esenciales de la sociedad si no que permite el acceso a varios servicios que quizá no sean estrictamente esenciales sino complementarios para varios procesos en el país" (NO1, 2020, p.2), además es tal la relevancia que tiene esta infraestructura, que se convierten en "blancos susceptibles de ataques" (Forigua Rojas, 2020, p.2).

Con lo analizado en el presente apartado, se vincula convincentemente explicaciones que pocos investigadores abordan académicamente en materia de protección de la Infraestructura Estratégica y su incidencia en los procesos sociales, evidenciando su importancia y su preservación o protección. Así como la analogía existente en el impacto social como parte de las transformaciones básicas de las comunidades, en ámbitos como el de infraestructura, economía, desarrollo y políticas públicas, entre otros. De acuerdo con estas consideraciones expuestas en el presente análisis, toma fuerza la afirmación que la presente investigación académica permitirá plantear una propuesta de intervención que redundará directamente en los beneficios finales de los servicios que este tipo de infraestructura sustenta en desarrollo de las funciones esenciales 
de la sociedad, convirtiendo este fenómeno estudiado en gran medida en un medio satisfactor para cubrir este tipo de necesidades.

Importancia del Sector Defensa en la protección de la infraestructura estratégica cómo infraestructura que sustenta los servicios esenciales: Revisando el anterior análisis se logra evidenciar la importancia de la protección de la infraestructura estratégica cómo infraestructura que sustenta los servicios esenciales, sin embargo, al realizar algunas reflexiones en estos apartados por parte de los expertos consultados, sobre la realidad de esta infraestructura vista desde una postura actual y desde el Sector Defensa, esa importancia que se evidenció en la presente investigación de manera teórica y a nivel internacional, se encuentra con procesos débiles, apenas vislumbrando algún tipo de principios que de por sí han tomado más tiempo del que se debía tomar y que desde luego han sido significativos pero insuficientes.De lo anterior, es necesario subrayar lo afirmado por el Director de Operaciones consultado, respecto a que "No existe actualmente una formulación de políticas de protección a la infraestructura estratégica en Colombia que aborde este tema específico"(NO1, 2020, p.2) y "no hay una identidad establecida y normada, cada sector de la infraestructura se ve como el único relevante, sin determinar una tabulación a nivel nacional por relevancia" según lo manifestado por el Consultor experto (ND3, 2020, p.2.)

Sin embargo, uno de los avances identificados en esta materia es el que resalta el Coordinador del Grupo de Infraestructura Crítica consultado, cuando indica que "el Plan Nacional de Desarrollo establece lineamientos en materia de protección de estas infraestructuras y se las asigna en sus lineamientos al Sector Defensa y este a su vez, establece en su Política de Seguridad y Defensa con acciones para velar por su protección" (ND2, 2020, p.3). A partir de estas afirmaciones, es importante dimensionar que los ejercicios que se enmarquen en temas de políticas públicas que, para la presente investigación en lo relacionado con la Protección de las Infraestructuras Estratégicas, deben contar con la participación público- privada de manera activa y permanente.

En ese sentido, el Dr. Forigua indica que "la formulación de la política pública ha estado más participativa", no obstante, "es reciente el proceso de contar con la participación ciudadana en la formulación de políticas públicas", porque "no hay una relación fuerte como se quisiera", y el Sector Defensa tiene una característica importante, y es que normalmente debe "responder sobre la marcha", así que los próximos ejercicios deben ir "vinculando a los sectores económicos" con el 
fin de lograr una construcción como "se ve institucionalizada en Europa y España" (Forigua Rojas, 2020, p. 3 - 4), pese a los "sesgos marcados que puedan tener" (NM1, 2020, p.2) como lo afirma la Asesora entrevistada.

Lo anterior, nos permite identificar una ventana de oportunidad que logra llevar de la teoría y los casos exitosos a nivel internacional, a la realidad. La importancia del Sector Defensa en la protección de la Infraestructura Estratégica, como infraestructura que sustenta los servicios esenciales, deja ver lo estrechamente interconectados que están con procesos socioeconómicos en el territorio, además porque requiere no solo su reconocimiento, sino lograr realizar acciones encaminadas a la obtención de resultados en materia de seguridad, que beneficiarán los diferentes sectores y con un impacto directo en la sociedad.

Identificación de Infraestructura Estratégica como prioridad en la Estrategia: Como ya se ha mencionado a lo largo del presente documento, la Protección de la Infraestructura Estratégica involucra elementos internos y externos e implica la destinación de recursos y capacidades por parte de las instituciones encargadas de su protección de acuerdo con los escenarios territoriales, sus factores y a la visión que se plantee como Estado para asumirla.

Visto el fenómeno desde la estrategia, es importante abordarlo desde los términos de gobernanza y seguridad. Los autores Alcaraz \& Zeadally se han referido a que la gobernanza, tiene que ver con los controles de seguridad (es decir, acciones) que se utilizan para administrar una organización. Los controles se definen en términos de políticas de seguridad, estándares, mejores prácticas y recomendaciones (Alcaraz \& Zeadally, 2015, p.59).

Con el fin de lograr identificar estas particularidades dentro de la estrategia actual estatal, se consultó a los expertos si estaban de acuerdo con que Colombia haya desarrollado las características descritas, en lo relacionado con la Protección de la Infraestructura Estratégica.

Por consiguiente, se identificó en lo conexo con el tema, que ha habido una evolución bastante importante, como lo indica el Dr. Forigua, pues a diferencia de otros años la "Gobernanza de choque utilizada en años pasados" ya no es una constante actual porque "se ha venido posesionando en la agenda pública", pese a que sigue siendo un "camino poco avanzado comparado 
internacionalmente". Asimismo, se han "dado pasos entorno a ello y entorno a un esquema de gobernanza cada vez más robusto", de hecho, "en los últimos documentos de política ha venido apareciendo con mayor fuerza lo relacionado con la Protección de Infraestructura Estratégica" debido a que "se ha pasado de un enfoque lineal a empezar a desarrollar características de la gobernanza" (Forigua Rojas, 2020, p.5-6). Sin embargo, uno de los retos es el identificado por la Asesora consultada, que implica que el "Estado deba llegar directamente a todas las regiones" porque en vista de "las condiciones económicas que tiene el Gobierno Nacional en este instante y la limitación de recursos, no ha sido posible llegar a todas las instancias del territorio colombiano" (NM1, 2020, p.3) y en ese sentido, el Coordinador del GIC particulariza en que "la gobernanza es una herramienta fundamental de integración entre todos los actores involucrados" (ND2, 2020, p.4).

Es así como se ratifica lo manifestado por los autores Fierro \& Mercado cuando indican la importancia de involucrar la utilización de perspectivas teóricas que puedan argumentar predictores de innovación organizativa (Fierro \& Mercado, 2012, p.95). De esta manera, cobra valor la relevancia de la planificación estratégica al interior de las organizaciones, con el fin de tener noción de su papel sobre los procesos para garantizar la seguridad y protección para la presente investigación, que conlleva a un posible sistema de Infraestructura Crítica como una solución que aporte a su protección, debido a que estos son concebidos inicialmente en este escenario y que contribuiría a plantear un contexto que permita entender que los Planes de Protección de las Infraestructuras Críticas hacen parte de una estrategia que se conciben y materializan en el marco de la adopción de esta.

Entonces, para entender lo referente a la protección de la Infraestructura Estratégica se han abordado diferentes contextos hasta el momento, tratando de evidenciar la lógica de su protección en beneficio de la sociedad y del ser humano sin dejar a un lado la relevancia política, económica y obviamente en lo referente a Defensa y Seguridad, en mira a involucrar lo citado por Fierro y Salgado sobre la estrategia de crecimiento la cual "implica un equilibrio entre la explotación de los recursos existentes y el desarrollo de nuevos, entendiéndose por recurso algo que podría considerarse como una fortaleza o debilidad de una empresa determinada"(Fierro \& Mercado, 2012,p.96). 
En tal sentido y con la intención de "formular el tema de manera un poco más estratégica" teniendo en cuenta que "el centro de lo estratégico es una visión un poco más de largo plazo, a partir de un diagnóstico que lleva implícito unas dinámicas de organización para que logre consolidar ese largo plazo" (Forigua Rojas, 2020, p. 16). Por consiguiente, implica que el Sector Defensa comience a planificar soluciones de tipo estratégicas que se puedan proyectar a corto, mediano y largo plazo para lo relacionado con la Protección de la Infraestructura Estratégica.

Aspectos generales de las infraestructuras estratégicas - infraestructuras críticas: Para abordar esta extensa subcategoría no era posible llegar a ella sin entender la relación de la Infraestructura Estratégica desde el punto de vista humano, como satisfactor y la relevancia que este tenga frente al acceso a los servicios esenciales de la sociedad, así como la relación directa que se debe plantear en términos de planeación y estrategia por el papel vital que estas juegan en la sociedad moderna como ya se ha explicado.

Ahora, el nuevo punto de partida será comprender, que para que el Sector Defensa de Colombia, pueda abordar cualquier tipo de escenario en materia de protección de las infraestructuras estratégicas, deben concebir su estrategia a partir de las particularidades propias del entorno. Es por ello, que se consultó al Grupo de expertos ¿Cuáles consideraban que son las particularidades que se relacionan con la Protección de los Activos Estratégicos de la Nación en un país como Colombia? Logrando plantear factores como: Las condiciones geográficas que hacen la diferencia en términos de protección, diversidad de amenazas y persistencias por parte de grupos al margen de la ley, grupos de crimen organizado, guerrilla residual, estructuras urbanas, delincuencia común, narcotráfico, dinámicas asimétricas, así como desigualdad social, entre otros que "muestran un desafío mayor para el Sector" (Forigua Rojas, 2020, p.7).

Respecto a lo relacionado con las fronteras, se convierte en un tema de Defensa Nacional que “influye en la Infraestructura Estratégica, porque puede haber también actores hostiles en el extranjero u otros países que eventualmente pueden tener intereses para que haya una coyuntura crítica en nuestro país y este tipo de infraestructura se consolida como un escenario" (Forigua Rojas, 2020, p.8).

Para sintetizar este punto, el Dr. Forigua habla de tres elementos, principales que particularizan la protección de los activos estratégicos: 1. El factor asimétrico que responde a las particularidades 
de Colombia. 2. El factor simétrico que responde a las visiones propias que manejan los ejércitos de cualquier país del mundo, para el caso de Colombia, la Fuerza Pública debe proteger sus Activos Estratégicos como parte de sus responsabilidades y 3 . Las particularidades que tenemos en las últimas décadas producto de la revolución tecnológica y demás, que estamos viviendo en este momento puesto que la interconexión de la infraestructura estratégica muestra una globalización de este fenómeno, porque "estos sistemas no son sistemas cerrados, no son sistemas aislados, obviamente son sistemas que están interconectados" (Forigua Rojas, 2020, p.8). Por lo anterior, se debe concebir que la Infraestructura Estratégica de la nación tenga que responder a esas particularidades (Forigua Rojas, 2020, p.8). Recapitulando, es preciso afirmar que "Combatir estas amenazas, que pueden poner en peligro la seguridad de los sistemas de control y sus infraestructuras críticas, se requiere que las estrategias de protección se diseñen cuidadosamente y se implementen adecuadamente" (Alcaraz \& Zeadally, 2015, p.55).

Como parte de comprender los aspectos generales de las infraestructuras estratégicas las Autoras Alcaraz y Zeadally manifiestan la importancia de considerar el nivel de dependencia entre recursos, componentes del sistema, funcionalidades y servicios ya que de acuerdo con el nivel de dependencia una afectación también puede causar un efecto cascada en el mismo sistema y en otras infraestructuras. Es así, como al respecto el Grupo de expertos consultados, nos permiten entender a través de sus afirmaciones que "la inversión en otros países y el posicionamiento del tema obedece al impacto en la seguridad nacional, el bienestar económico, la salud y la seguridad pública que una interrupción podría tener" y "los impactos deben considerarse en las proyecciones a futuro que se realizan" ya que en coyunturas críticas como la actual (Covic-19), "el impacto económico que tuvo esta pandemia, llevo a ver el sector salud, como un sistema interconectado, con una correlación tan clara y densa" (Forigua Rojas, 2020, p.13). Lo que refuerza "la importancia que tiene este tema en la agenda de los países (Forigua Rojas, 2020, p.10 -11 -12).

Es así como "cualquier factor que influya en la Infraestructura Crítica, definitivamente impacta en estos factores de atención" (NM1, 2020, p 4) y "cualquier interrupción física o virtual afectará y generará un impacto negativo en el desarrollo económico y en la continuidad de las operaciones de cualquier actividad económica" (ND2, 2020, p.5) de ello resulta necesario decir que a "nivel mundial tiene una gran relevancia el tema de la protección de Infraestructura Crítica, en el entorno 
cibernético y es una de las aristas que a futuro el Gobierno Nacional también debe empezar a impulsar" (ND2, 2020, p.5).

En este punto, se solicitó a los expertos sus deferencias respecto a si consideran que ¿comprender el poder económico y político de las infraestructuras estratégicas, es esencial para que sean justas, equitativas y sostenibles?, en tal sentido el Dr. Forigua manifiesta que:

"es supremamente importante que de esto se tenga una muy buena comprensión, obviamente de sus connotaciones económicas y políticas, porque por desconocimiento se podría considerar que este tema en principio no es tan relevante, es decir, si yo no tengo muy claro la relevancia de ese tema, muy probablemente no alcanzo a entender las interconexiones económicas y políticas que tiene. Se requiere saber del tema para generar consenso político, por la naturaleza del Sector Defensa se tiende a tener una visión un poco más clara" (Forigua Rojas, 2020, p.10).

Unido a esta afirmación el Coordinador GIC indica que al brindar a la "sociedad, desarrollo económico el país puede incentivar a futuro todo el tema de inversión, que redundaría en el bienestar para la misma sociedad" y complementa lo indicado por la Asesora respecto a qué estas afırmaciones permiten "determinar cuál va a ser nuestro nivel de responsabilidad para contribuir a que estos factores se den en la comunidad y cómo vamos a llegar a ellos".

Sin embargo, pese a estas declaraciones el Consultor indica que "debemos entender esta infraestructura como la resultante final de una política justa, imparcial y bien ejecutada" sumado a ello el Dr. Forigua ratifica que para que las infraestructuras sean vistas como equitativas, justas y sostenibles, hay que vincularlas a la discusión pública con mucha más claridad y esto amerita a verlas como un sistema con muchos elementos que están interconectados, pues cuando se mira si las interconexiones económicas y políticas pueden inferir en una serie de conclusiones que indican puntualmente poder realizar un diagnóstico de cómo se está gestionando la infraestructura estratégica, también se está haciendo un ejercicio, precisamente para lograr esos términos que no siempre dan cuenta de una prestación de servicios equitativos, justos, o sostenibles (Forigua Rojas, 2020, p.10-11). En tal sentido, se puede inferir que efectivamente el Sector Defensa debe 
propender por desarrollar estas actividades identificadas lo que permitirá contribuir a aspectos socioeconómicos de la mayor relevancia.

En este sentido ya se logra decantar la idea orientadora del presente documento, cuando se afirma que es posible que existan aspectos fundamentales que se pueden considerar en el contexto del Sector Defensa para que se contribuya en la Protección de la Infraestructura Estratégica a partir de la estrategia de Protección de la Infraestructura Crítica adoptada por España. Por consiguiente, se indagó al Grupo de Expertos si ¿Debería el Sector Defensa considerar estrategias encaminadas a un sistema de gestión normalizado para la protección de Infraestructura Estratégico o Crítica (Activos Estratégicos de la Nación)? Y del cual se obtuvo el siguiente resultado:

- Se debería considerar un Sistema de Gestión Normalizado, como parte de las herramientas que se han venido utilizando y que lleve a una aproximación mucho más formal a la trabajada en el Sector Defensa, las cuales han sido enmarcadas en políticas y planes sectoriales que no han tenido un enfoque lo suficientemente sistémico (Forigua Rojas, 2020, p.11-12 - 13 -14).

- Cobra valor la definición de sistema que efectivamente se puede ver desde el Sector Defensa, pero se debe considerar verlo como un SUBSISTEMA que hace parte de un gran sistema, porque esto incluye infraestructura de otros sectores, así como la participación de otros actores a nivel estatal y sectorial, lo que complejiza el nivel de avance para establecer las características del sistema, siendo esto lo que marcará las distintas velocidades en el momento de desarrollar lo correspondiente al Sistema Nacional (Forigua Rojas, 2020, p.11-12 - 13 -14).

- Por la vocación operativa, el MDN debe tener bajo su responsabilidad una parte del sistema, este sector no alcanzaría a responder por todo el sistema, pese a que tiene la posibilidad de moverse a ritmos distintos, por la vocación operacional ya que su estructura está pensada para operar, sin embargo, no debería recaer la absoluta responsabilidad de este sistema de gestión (Forigua Rojas, 2020, p.11-12 - 13 -14).

- Es importante establecer estándares mínimos en materia de Protección de la Infraestructura físicas (ND2, 2020.p.6) que normalice esta protección porque la infraestructura estratégica o crítica hace parte del desarrollo natural del país y sin este desarrollo no hay evolución (ND3, 2020, p.5.) 
- Cuando se hace referencia a un sistema de gestión quiere decir que ahí se está gestionando algo y varias cosas que están interconectadas. Un Sistema de Gestión Normalizado si ayudaría un poco a la integralización. La Protección de la Infraestructura Estratégica, requiere de darle la integralidad que necesita abarcar el tema y por ende en algún momento requerirá ser visto desde Presidencia de la República (Forigua Rojas, 2020, p.11-12 - 13 -14).

- A nivel mundial los Gobiernos han venido adelantando o adoptando esquemas de protección de manera estandarizada. En ese sentido, Colombia tiene que considerar la cooperación internacional quizá a través de la articulación con los Gobiernos que han tenido avances en los temas de protección de infraestructura, para poder imitar las mejores prácticas y generar nuevas acciones en materia de protocolos de seguridad para la protección de infraestructuras físicas. (ND2, 2020.p.6)

Una vez descrito lo anterior, se le solicitó al Grupo de expertos que jerarquice las labores que actualmente realiza el Sector Defensa para la Protección de la Infraestructura Estratégica de Colombia de acuerdo con el nivel intervención que normalmente identifica que realiza. Donde cinco (5) es la labor que hace normalmente, tres (3) es la labor que ocasionalmente hace y uno (1) es la labor que rara vez asume o hace con menor frecuencia.

Al respecto, de manera unánime se jerarquizó: la reacción (5), la detección y respuesta (3) y la prevención (1). Lo que permite deducir que se requiere incentivar estrategias, planes, programas, proyectos y acciones que permitan al Sector Defensa plantear escenarios que contribuyan a abordar las labores de prevención antes que las de reacción.

En consecuencia a lo expuesto en este apartado, esta subcategoría permite particularizar que una de las alternativas de intervención que el Sector Defensa podría adoptar es plantear una estrategia de Protección de la Infraestructura Estratégica, que pueda materializarse a través de la creación, gestión y consolidación de un Subsistema Sectorial que a mediano o largo plazo haga parte de un Sistema Nacional de Infraestructura que sea gestionado desde el Gobierno Nacional, que dimensione algunas características que permitan mejorar las labores que actualmente ejerce la Fuerza Pública desde su ámbito de responsabilidad. 
De esta forma, es que se podría lograr planificar el Subsistema que debería gestionar el Sector y que ayudará a mejorar lo relacionado con la toma de decisiones que a su vez disminuirá varios escenarios de incertidumbre y reacción, mejorando los de detección y prevención, permitiendo una reorganización o gestión de recursos y responsabilidades en lo relacionado a la Protección de la Infraestructura Estratégica, que a su vez tendrá un impacto socialmente significativo en los territorios, mostrando un avance a nivel de política pública y vinculando cada vez más actores con el fin de hacer un proceso mucho más formal que permita abarcar ese sistema complejo que es la Infraestructura Crítica del país.

Manejo de características relativamente generales de un Plan de Protección de Infraestructura Crítica, teniendo como referente algunos elementos de la Estrategia de Protección de Infraestructura Crítica de España: Como teóricamente ya fue explicado en el presente documento, en anteriores apartes, España administra la Protección de la Infraestructura Crítica a través de dos herramientas principales, la primera el Sistema de Protección de Infraestructuras Críticas y la segunda, el Centro Nacional de Protección de Infraestructura Crítica, que permiten dimensionar la gestión tanto de la protección como las amenazas desde un enfoque sistémico.

Es por ello por lo que se logró determinar la importancia de indagar sobre el concepto de colaboración público - privada, la identificación de la infraestructura estratégica, la categorización de la Infraestructura Crítica, la identificación y administración de los riesgos, la identificación de los agentes del sistema y lo relacionado con la resiliencia, de forma tal que a continuación se relacionan los principales apartes expuestos por el Grupo de expertos consultados sobre estas características que definitivamente se deberán considerar en la propuesta de intervención que se plantee.

Colaboración Público - Privada: Existen espacios de interlocución entre el Sector Defensa y el sector público - privado normalmente dentro de parámetros específicos y coyunturales, no dentro de una visión estratégica. Sin embargo, para el sector no es un tema que se tenga que posesionar desde cero, pero que se podría robustecer, a partir de darle una visión mucho más estratégica, de largo plazo, con una mayor proyección, para no caer en la coyuntura (Forigua Rojas, 2020, p.16 -17). 
Es importante considerar que hay que establecer procesos o protocolos de corresponsabilidad o de responsabilidad compartida con el sector privado, de hecho, el sector privado debería desarrollar mejores procesos de autoprotección de sus infraestructuras y complementarlas con las acciones que presta el Gobierno Nacional a través del Sector Defensa (ND2, 2020, p.5-6) sin abandonar la responsabilidad propia que tiene la Fuerza Pública en su protección (ND3, 2020, p.7.).

Se deberían establecer canales más directos que permitan una interacción más activa, receptiva de doble vía, con escenarios puntuales para gestionar la protección de la infraestructura estratégica y que parametrice compromisos no solo por parte del estado sino por parte de estos actores de acuerdo con su responsabilidad (NO1, 2020, p.6).

Los Proyectos de Interés Nacional y Estratégico (PINES), deben hacer parte de la infraestructura estratégica y contar con la catalogación correspondiente que lo clasifique como Infraestructura Crítica (NM1, 2020, p.5-6).

Lo primero que se identifica de la consulta de expertos, es que el nivel directivo coincide en que el Sector Defensa tiene la infraestructura estratégica identificada bajo los parámetros que la Fuerza Pública ha venido determinando y de acuerdo con la importancia que esta tenga para el sector o de acuerdo con características que le den la relevancia, las cuales son normalmente coyunturales. En sentido opuesto, el nivel medio y operativo consultado indican que el Sector Defensa no tiene la Infraestructura Estratégica identificada formalmente con una misma línea de levantamiento que permita su agrupación, por así decirlo, a través de un catálogo de infraestructuras físicas. En tal sentido, a continuación, se relacionan algunas conclusiones de este apartado que es importante conocerlas y considerarlas.

El Sector Defensa si cuenta con la identificación de la infraestructura estratégica, sin embargo, se puede considerar que hace parte de información reservada, lo que si se pudiera afirmar es que no se encuentra formalmente dentro de un catálogo de infraestructuras físicas y esto a su vez, no permite hablar en términos de una catalogación (Forigua Rojas, 2020, p.17).

Lo anterior, teniendo en cuenta que el Ministerio de Defensa Nacional, cuentan con criterios de seguridad que permiten determinar características de las amenazas de manera sectorial, pero 
que requieren adicionar los criterios técnicos del sector que permita determinar su catalogación, porque actualmente se trabaja en función de la amenaza y la amenaza, define muchos de sus criterios casi que de manera territorial, lo que puede ser un blanco para los grupos al margen de la ley y convirtiéndose en una prioridad para el sector y la Fuerza Pública. Cuando se busca unificar la información bajo el concepto de país, es cuando recobra importancia verificar los criterios para realizar este ejercicio, el cual debe iniciar por la determinación de los sectores que se van a abordar y la discusión de criterios que permitiera esta formalización (Forigua Rojas, 2020, p.17).

La determinación clara y especifica de criterios objetivos, advierten que no se subjetivasen los diálogos y el desarrollo del ejercicio. Aunque sectorialmente se pueda contar con algunos de ellos, al intentar abordar todo en un solo contexto se podría afirmar que no sabemos exactamente o muy bien de lo que estamos hablando, por no contar con esos criterios orientadores. Y por la coyuntura en la que normalmente el sector debe maniobrar, en este sentido, el criterio es lo que permitirá catalogar y categorizar la infraestructura estratégica, lo que requiere lograr construir un poco de distancia con la coyuntura y posicionar una visión más estratégica del tema (Forigua Rojas, 2020, p.17).

- Identificación y administración de los riesgos y agentes del sistema: Este punto está directamente relacionado con el anterior, basados en las percepciones de los diferentes niveles, sin embargo, se resaltan las principales afirmaciones obtenidas por parte del grupo de expertos que contribuyen a esta clasificación en materia de riesgos y agentes del Sistema de Protección de Infraestructura Críticas, PIC, quienes indican que:

- El Sector Defensa no tiene identificado los agentes del sistema que deberían hacer parte del Sistema PIC, cuyas principales responsabilidades están relacionadas con el correcto funcionamiento de los servicios esenciales. De hecho, no se cuenta con la determinación exacta de que es un servicio esencial, lo que no permite que se asocie fácilmente el rol del Sector Defensa y al sistema PIC. 
- Lo contrario ocurre en lo relacionado con la seguridad de los ciudadanos que también contempla el Sistema PIC, ya que en el Sector Defensa si identifica fácilmente su nivel de responsabilidad y en tal sentido, como se ha venido evidenciando en la presente investigación, se podría hablar de que garantizar la seguridad ciudadana hace parte de un servicio esencial (Forigua Rojas, 2020, p.17 - 24).

- Se debe ampliar la participación a otros actores como el del sector privado y buscar contar con la participación y cooperación internacional, con el fin de ampliar la visión frente a cuáles son las mejores prácticas que se deban implementar para la protección de esta infraestructura (ND2, 2020, p. 7 - 8).

Resiliencia: Hablar de resiliencia es hablar de uno de los atributos de los sistemas como lo afirma el Dr. Forigua, es por ello que vale la pena enunciar algunos aportes realizados por el Grupo de Expertos al respecto y a quienes se les preguntó por individual ¿Cree usted que el Sector Defensa es resiliente ante las afectaciones de los Activos Estratégicos de la Nación?

- La resiliencia reafirma que España claramente cuenta y administra la infraestructura estratégica y crítica a través de un sistema, porque la resiliencia es un atributo sistémico junto con la autoorganización y la jerarquía (Forigua Rojas, 2020, p. 24 -27).

- El Ministerio de Defensa si es bastante resiliente como sector por estar inmerso en dinámicas de fuerza, valga decir lejos de un óptimo nivel, pero incluso más resilientes que las demás entidades el Estado. Sin embargo, hay un camino largo, como parte del ejercicio de las capacidades propuesto por la OTAN que buscan una resiliencia cada vez mayor (Forigua Rojas, 2020, p. 24 -27).

- La resiliencia del Sector Defensa es de un Subsistema de un sistema mucho más complejo. Al mirar globalemente lo que debería ser el Sistema Nacional, muy probablemente no sale tan resiliente, porque sería una sumatoria de subsistemas y no hay como saberlo actualmente, ya que se está infiriendo esto de manera especulativa porque no se tienen criterios objetivos establecidos (Forigua Rojas, 2020, p. 24 -27). 
- El Sector Defensa es resiliente en gran medida, ya que tiene la capacidad de recuperarse muy rápido cuando sucede una afectación a la infraestructura y esto se logra a través de las mejores prácticas y lecciones aprendidas. La resiliencia se ha aplicado frente a acciones oportunas para poder garantizar la operación de esta infraestructura estratégica (ND2, 2020, p.9).

- Es un sector que vive abordando diversas problemáticas que requieren de una autogestión para abordarlas, ya sea en recursos, en métodos o inclusive en estrategias que han permitido la atención y recuperación de este tipo de infraestructuras (NO1, 2020, p.1).

- Los factores de riesgo que tiene el Sector generan el tener que ser más reactivos que preventivos (NM1, 2020, p.8). Porque a pesar de contar con la capacidad de superar las diferentes circunstancias que se presentan, normalmente se realizan bajo la marcha (ND3, 2020, p.8).

Elementos de la Estrategia de Protección de Infraestructura Crítica de España como referente.

En consecuencia al desarrollo de toda la investigación realizada y con base a la experiencia compartida por el Grupo de expertos que participaron en la presente investigación, desde el rol de investigadora y después de analizar la información recolectada, en vista que la Estrategia de Protección de la Infraestructura Crítica adoptada por España ha sido considerada para el presente documento como un referente, se procede posteriormente a identificar a través de la Lista de Chequeo las características extraídas que servirán para verificar la presencia o ausencia de estos ítems en el contexto del Sector Defensa de Colombia, frente a lo referente a la protección de infraestructura estratégica. Es de aclarar que como su objetivo no es estadístico, los criterios identificados en ella podrán ser tenidos en cuenta a la hora de realizar la propuesta de intervención como parte de las lecciones aprendidas de España.

Las características registradas en la Lista de Chequeo son veinte (20), las cuales agrupan cuarenta y cuatro (44) ítems en total, de ellas solo tres categorías enlistadas en los numerales 4, 8 y 9 muestran algún tipo de avance por parte del Sector de Defensa en comparación con España, como se observa en la tabla adjunta. 
Tabla 1. Ítems de avance Lista de Chequeo

\begin{tabular}{|c|l|c|c|c|}
\hline N & \multicolumn{1}{|c|}{ DESCRIPCIÓN } & SI & NO & OBSERVACIONES \\
\hline 4 & $\begin{array}{l}\text { Se ha involucrado la acción colaborativa del sector } \\
\text { privado, basada en el concepto de la cooperación } \\
\text { público-privada y en el intercambio de información } \\
\text { sensible }\end{array}$ & $x$ & & $\begin{array}{l}\text { Parcialmente, cuando el operador requiere } \\
\text { intervención por parte de la Fuerza Pública }\end{array}$ \\
\hline 8 & $\begin{array}{l}\text { Se promueve la existencia de una cultura de } \\
\text { seguridad de las infraestructuras Críticas en el } \\
\text { ámbito de las Administraciones Públicas. }\end{array}$ & $\times$ & & Ocasionalmete y es mas de tipo circunstacial \\
\hline 9 & $\begin{array}{l}\text { La Protección de la Infraestrctura Crítica está } \\
\text { subordinada a la Política de Seguridad y Defensa } \\
\text { Nacional. }\end{array}$ & $\times$ & & $\begin{array}{l}\text { Se contempla dentro de la Política, aunque no } \\
\text { se ha dimensionado dentro de un plan Nacional } \\
\text { de Protección de Infraestrctura Crítica. }\end{array}$ \\
\hline
\end{tabular}

Fuente: elaboración propia

De acuerdo con lo indicado por el Dr. Forigua, existen espacios de interlocución entre el Sector Defensa y el sector público - privado normalmente dentro de parámetros específicos y coyunturales, pero no dentro de una visión estratégica (Forigua Rojas, 2020, p. 15), por lo que "podría delimitar las relaciones en escenarios de apagar incendios" (Forigua Rojas, 2020, p. 15).

De los cuarenta y un (41) ítems restantes en el numeral doce (12), solo ocho (8) de ellos cuentan con algunas particularidades, pese a que no cumplen con la descripción del ítem y los treinta y tres (33) ítems restantes, registran el no cumplimiento con su descripción que, en vista del avance del Sector Defensa ya detallado, se convierten en puntos de referencia para ser asumidos en desarrollo de la Protección de las Infraestructuras Estratégicas, como ventanas de oportunidad para mejorar este proceso. Como lo enunciado por el Coordinador del GIC quien resalta que "se debe ampliar la participación a otros actores como la del sector privado y porque no el de ampliar la cooperación internacional" (ND2, 2020, p.8). 
Tabla 2. Ítems negativos de Lista de Chequeo con observaciones

\begin{tabular}{|c|c|c|c|c|}
\hline $\mathrm{N}$ & DESCRIPCIÓN & SI & NO & OBSERVACIONES \\
\hline \multirow{8}{*}{12} & Principios de Coordinación & & & $\begin{array}{l}\text { Únicamente en casos particulares } \\
\text { eindividuales }\end{array}$ \\
\hline & $\begin{array}{l}\text { Se ha establecido lo relacionado con la intervención } \\
\text { del Estado y la competencia departamental. }\end{array}$ & & $x$ & $\begin{array}{l}\text { No direccionado a la Protección de la } \\
\text { Infraestrctura Crítica, únicamente desde el } \\
\text { marco legislativo general como el Plan de } \\
\text { Desarrollo Nacional. }\end{array}$ \\
\hline & $\begin{array}{l}\text { Se ha identificado responsabilidad compartida y } \\
\text { cooperación público privada. }\end{array}$ & & $x$ & $\begin{array}{l}\text { Únicamente en casos particulares e } \\
\text { individuales. no es una constante. }\end{array}$ \\
\hline & $\begin{array}{l}\text { Se ha identificado líneas de acción que contribuyen } \\
\text { con el Equilibrio y Eficiencia conjunta público - privada } \\
\text { de las Infraestructuras Estratégicas }\end{array}$ & & $x$ & $\begin{array}{l}\text { Iniciativas aisladas por parte de la Fuerza } \\
\text { Pública, normalmente en términos reactivos }\end{array}$ \\
\hline & $\begin{array}{l}\text { Se ha direccionado las acciones de Protección de la } \\
\text { Infraestructura Crítica desde un enfoque integral. }\end{array}$ & & $x$ & Actualmente no se cuenta con un PICN \\
\hline & $\begin{array}{l}\text { Se ha establecido un marco regulatorio común } \\
\text { y homogéneo, que responda a la Protección de } \\
\text { Infraestructura Críticas. }\end{array}$ & & $x$ & $\begin{array}{l}\text { Sectorizado, más en líneas generales en } \\
\text { tiempos contractuales. }\end{array}$ \\
\hline & $\begin{array}{l}\text { Se realiza una planificación escalonada que permita } \\
\text { identificar, evaluar, prevenir y mitigar los riesgos }\end{array}$ & & $x$ & $\begin{array}{l}\text { Únicamente en casos particulares e } \\
\text { individuales, de manera coyuntural }\end{array}$ \\
\hline & $\begin{array}{l}\text { Se concibe la Resilencia dentro de la Protección de las } \\
\text { Infraestructuras Críticas. }\end{array}$ & & $x$ & Ocasionalmente de manera coyuntural \\
\hline
\end{tabular}

Fuente: elaboración propia

Finalmente, en vista de los resultados obtenidos posterior a la aplicación de los instrumentos determinados, se le realizó una última solicitud al Grupo de Expertos, consistente en indicar ¿Qué debería considerar el Sector Defensa para fortalecer la Protección de los Activos Estratégicos de la Nación?, con lo que se relacionan las siguientes apreciaciones más relevantes:

- Manejar el tema de una manera más estratégica (Forigua Rojas, 2020, p. 24).

- Considerar el apoyo y cooperación de gobiernos internacionales para fortalecer las acciones de Protección de las Infraestructuras Estratégicas e incluir en la agenda del Gobierno Nacional, todos los aspectos inherentes en materia de protección, prevención, y detección de amenazas de manera integral (ND2, 2020, p.8). 
- Realizar la categorización de la Infraestructura Crítica e identificar y visualizar los riesgos inherentes a ella, para generar acciones que conlleven a evitar que se materialicen (NM1, 2020, p.8).

- Establecer el sistema de protección de las Infraestructuras Críticas que agrupe sectores y cuente con los apartados que se requieran para desarrollar esta labor de manera coordinada, logrando hacer una directriz de obligatorio cumplimiento en el que confluyan los actores involucrados en su administración, control y preservación (ND3, 2020, p.8).

Normalizar la protección desde una estrategia unificada a través de un Sistema que direccione características, responsabilidades, estándares y en si reglas de juego con la colaboración público privada y entidades del Estado (NO1, 2020, p.7).

Una vez abordado el fenómeno de Protección a la Infraestructura Estratégica a través del desarrollo de la presente investigación donde se logró acotar los objetivos propuestos, se presentan las reflexiones finales y el apartado correspondiente a la propuesta de intervención.

\section{PROPUESTA DE INTERVENCIÓN}

En virtud de poder desarrollar lo relacionado al último objetivo específico planteado que habla de proponer líneas de acción que contribuyan al Sector Defensa a fortalecer la Protección de los Activos Estratégicos de la Nación y como resultado de la investigación realizada, se plantea la posibilidad de realizar la Versión 1 del Catálogo de Infraestructura Crítica Física por parte del Sector Defensa, como Subsistema del Sistema Nacional de Protección que se debería concebir a futuro y que permitirá convertirse en los primeros pasos que pueda dar el sector en miras a generar estrategias que contribuyan a su protección y que se adapten a las tendencias internacionales.

Cabe aclarar que el resultado de la investigación por si sola, ha identificado entre otros aspectos, que este es un tema de largo alcance dentro del Sector Defensa que amerita trabajarlo en diferentes fases y momentos, de acuerdo a los avances que se vayan obteniendo en esta materia, lo cual permite determinar que incluso por no ser un tema abordado de manera estandarizada por la Fuerza Pública, debería concebirse en que cada etapa debe ser dinámica y que recoja no solo las lecciones aprendidas de las fases que se logren desarrollar, sino que se vaya ajustando en la medida que se logre avanzar en cada sector y a su vez, en la medida en que se vayan integrando 
los diferentes actores involucrados que tienen injerencia en lo relacionado a la Protección de la Infraestructura Estratégica y Crítica.

\section{CONCLUSIONES}

- La Infraestructura Estratégica es una de las principales herramientas de un país para el sostenimiento fiscal del mismo.

- Existe una relación evidente entre la Infraestructura Estratégica y las necesidades básicas humanas, así como su satisfacción a través de ella.

- El Sector Defensa requiere repensar el proceso de protección de la Infraestructura Estratégica a partir de una proyección a corto, mediano y largo plazo.

- Para concebir un Sistema de Protección de Infraestructura como el planteado internacionalmente y para la presente investigación el establecido por España, desde el Sector Defensa, es necesario pensarlo como un Subsistema que hace parte de un Sistema Nacional que necesariamente involucraría un número importante de instituciones estatales y que requiere contar de la participación público privada.

- La magnitud de un Sistema Nacional de Protección es el camino por seguir, pero en un escenario a largo plazo y efectivamente requiere de voluntad política y del liderazgo del Gobierno Nacional.

- El Sector Defensa debe empezar a identificar escenarios que le permitan gestionar de una manera más acertada lo relacionado con la Infraestructura Estratégica visto desde sus posibilidades, con el fin de intentar movilizar los agentes intervinientes en la administración, protección y gestión de estas. Es por ello, por lo que una de las iniciativas que cobraría valor y por ser aparentemente de los primeros pasos no sería de menor importancia, es la de realizar el Primer Catálogo de Infraestructura Crítica Física el cual se deberá concebir abordando un sector económico a su vez, con el fin de evitar complejizar el ejercicio. 
- Hablar del Primer Catálogo de Infraestructura Crítica Física, necesariamente permite identificar que podría estar liderado por el Sector Defensa, pero adicionalmente concibe realizar procesos relacionados con conceptos organizacionales como asignación de responsabilidades, identificación de dinámicas de coordinación, jerarquía, asignación de recursos territoriales, interacción público privada, innovación tecnológica en el proceso de protección, interoperabilidad, caracterización, robustecimiento de recursos, identificación y administración de riesgos, cooperación internacional y lo relacionado con la sostenibilidad entre otros.

- Los sectores públicos - privados necesitan con urgencia la delimitación de un glosario que compendie términos necesarios para entender la dinámica de las Infraestructuras Estratégicas y lo que involucra su protección.

- Los sectores públicos privado deben propender para que los ciudadanos del común logren entender la importancia que representa la Infraestructura Estratégica y con ello lograr resignificar su impacto en el desarrollo social de los territorios.

- Es importante considerar las lecciones aprendidas y los casos de éxito que permitan un intercambio de información frente a la Protección de la Infraestructura Estratégica para adaptarlo a nivel sectorial y estatal según corresponda.

- Se debe establecer criterios orientadores que permitan eliminar la subjetividad en los diferentes escenarios.

- El objetivo principal de la protección de las infraestructuras debe estar centrado en la característica de oportunidad que permita, entre otros aspectos, implementar, mejorar o sostener las medidas de seguridad necesarias de acuerdo con las perspectivas de cada sector, con el fin de buscar la integralidad de estas.

- Se debe considerar revisar la presente estrategia que el Sector Defensa está implementando para la protección de la Infraestructura Estratégica, teniendo en cuenta que esta debe adaptarse a los nuevos desafíos o a las dinámicas del entorno. 
- La tecnología debe estar involucrada en todos los escenarios relacionados con la protección de la Infraestructura Estratégica dimensionados a corto, mediano y largo plazo impulsando nuevas concepciones de transformación de la seguridad.

- En vista que un gran porcentaje de la Infraestructura Estratégica que sustenta los servicios esenciales está bajo la responsabilidad de operadores privados, por su administración u operación, es fundamental desarrollar líneas de acción que permita asumir una colaboración público privada que beneficie los intereses de los involucrados y en especial el de la sociedad.

- La Fuerza Pública es la ejecutora de toda la estrategia y tiene la responsabilidad que se encuentra en el Sector Defensa, por lo tanto, se debe comenzar a socializar con los comandantes y las unidades lo relacionado a entender la Infraestructura Estratégica.

- Se debe concebir una gestión adecuada de recursos.

- Existe una relación muy fuerte entre la Infraestructura Estratégica, el papel del Estado, los sectores económicos y la sociedad civil frente a las dinámicas propias de esta infraestructura y los modelos relacionales que sustenta su cooperación, operacionalización y beneficio de ellas de allí la importancia de redefinir políticas, priorizar programas e incluso modificar presupuestos.

- De acuerdo con lo teorizado por Neef y el desarrollo de la investigación los responsables de las Infraestructuras Estratégicas deben proponerse entablar una relación cercana entre ellas y la sociedad para procurar cambios decisivos y repensados, tanto en los servicios que brindan como en los beneficios que se reciben de su parte, con el propósito que ambas partes cuenten con una mirada de tipo empresarial, holística, consciente y más humana de su papel y su participación en lo relacionado a la protección de determinada infraestructura en un sector territorial especifico.

- Se sugiere para futuras investigaciones sobre protección de la Infraestructura Estratégica, concebir una categoría relacionada con planeación basada en capacidades, recursos, sostenibilidad, prospectiva estratégica y concepción sistemática de las organizaciones e innovación. 


\section{REFERENCIAS}

Abreu, J. L. (2014). El Método de la Investigación Research Method. Daena: International Journal of Good Conscience, 9(3), 195-204.

Aims \& scope - Energy Research \& Social Science IScienceDirect.com. (s. f.). https://www.sciencedirect. com/journal/energy-research-and-social-science/about/aims-and-scope

Alcaraz, C., \& Zeadally, S. (2015). Critical infrastructure protection: Requirements and challenges for the 21st century. International Journal of Critical Infrastructure Protection, 8, 53-66. https://doi. org/10.1016/j.jjcip.2014.12.002

Amutio, M. A., Candau, J., \& Mañas, J. A. (2012). MAGERIT - versión 3.0. Metodología de Análisis y Gestión de Riesgos de los Sistemas de Información. Libro I - Método. En S. G. Técnica (Ed.), Ministerio de Hacienda y Administraciones Públicas (Ministerio, Vol. 2006, Números 630-12-1718). Centro de Publicaciones Gobierno de España.

Becerra, O. E. (2012). Elaboración de Instrumentos de Investigación. Departamento de investigación del CUAM Caracas.

Blaxter, L., Hughes, C., \& Malcolm, T. (2002). Cómo se hace una investigación (2.a ed.). Gedisa.

Bolseguí, M., \& Fuguet Smith, A. (2006). Construcción de un modelo conceptual a través de la investigación cualitativa. Sapiens: Revista Universitaria de Investigación, 7(1), 207-230.

Buendía Eisman, L., Colás Bravo, P., \& Hernández Pina, F. (1998). Métodos de Investigación en Psicopedagogía (C. Casado Lumbreras (Ed.); Mcgraw-HIL). Mcgraw-HILL/INTERAMERICANA DE ESPAÑA, S. A. U.

Canale, A., \& Martín, M. (s. f.). Gobernabilidad y Seguridad en América Latina: Desafíos del sector Defensa. Universidad Santiago de Chile. 
Centro de Ciberseguridad Industrial. (2013). La Protección de Infraestructuras Críticas y la Ciberseguridad Industrial. (1.a ed.). Centro de Ciberseguridad Industrial. https://www.cci-es.org/ documents/10694/43057/Documento+Presentación+PIC+y+Cl/8805e448-bc21-47bb-b287bc774382696d

Colás Bravo, P., \& De Pablos Pons, J. (2012). Aplicaciones de las tecnologías de la información y la comunicación en la investigación cualitativa. Revista Espanola de Pedagogia, 70(251), 77-92.

Correa, G. J., \& Yusta, J. M. (2014). Planes de Protección de Infraestructuras Críticas Critical Infrastructure Protection Plans. ResearchGate, September. https://doi.org/10.13140/2.1.2838.4642

Díaz-Bravo, L., Torruco-García, U., Martínez-Hernández, M., \& Varela-Ruiz, M. (2013). La entrevista, recurso flexible y dinámico. Investigación en Educación Médica, 2(7), 162-167. https://doi. org/10.1016/s2007-5057(13)72706-6

Drucker, P. F., Management, D., Sociales, C., \& Marie, G. (1999). Management - Automanagement por Peter Drucker.

Elliott, J. (s.f.). El cambio educativo desde la investigación acción. https://books.google.com.co/ books?hl=en\&lr=\&id=6cl-VsOF6isC\&oi=fnd\&pg=PA13\&dq=ELLIOTT,+J.+(1993).+El+cambio+ educativo+desde+la+investigación-acción,+Madrid:+Morata.\&ots=YhFalOvm_e\&sig=kgctCOgOMLCArc7AaTg8AH5Yfo\&redir_esc=y\#v=onepage\&q=el estudio de una $s$

Entrevista. (s. f.). usecnetwork, 80-87.

Estrategia de Seguridad Nacional 2017 (Presidenci). (2017). Imprenta Boletín Oficial del Estado.

Fierro, M. E., \& Mercado, S. P. (2012). La innovación organizativa y sus predictores desde la Teoría de Recursos y Capacidades. Administración y Organizaciones, 15(28), 93-115.

Forigua Rojas, E. (2020). ND1 Entrevista Experto. 
Galindo Sierra, F. J. (2016). Protección de Infraestructuras Críticas: Un Análisis De Derecho. En Universidad de Málaga.

Gutiérrez Pérez, J., Pozo Llorente, T., \& Fernández Cano, A. (2002). Los estudios de caso en la lógica de la investigación interpretativa. Arbor, 171(675), 533-557. https://doi.org/10.3989/arbor.2002. i675.1045

Hernandez Sampieri, R., Fernández Collado, C., \& Butista Lucio, P. (2004). Metodología de la Investigación. En A. Martínez (Ed.), Physical Review B. McGraw-Hill Interamericana.

Historia - Ministerio de Defensa Nacional de la República de Colombia. (s. f.). https://www. mindefensa.gov.co/irj/portal/Mindefensa/contenido?NavigationTarget=navurl://1494c44e $2596646 \mathrm{~d} 35 \mathrm{f} 4060084 \mathrm{fd} 9 \mathrm{b0} 2$

INFOR. (2016). Memoria 2016. https://www.infor.cl/images/pdf/MEMORIA_2018.pdf, 94. http://www. datosdelanzarote.com/itemDetalles.asp?idFamilia=18\&idltem=7589

Jefatura del Estado. (2011). Ley 8/2011, de 28 de abril, por la que se establecen medidas para la protección de las infraestructuras críticas (pp. 1-11). Rey de España. https://www.boe.es/buscar/ act.php?id=BOE-A-2011-7630

Manfred, M.-N., Antono, E., \& Martín, H. (2010). Desarrollo a Escala Humana una opción para el futuro. En CEPAUR Fundación Dag Hammarskjold (Vol. 36, Número 142).

Maya, E. (2014). Métodos y Técnicas de investigación. En Familia. Revista de Ciencias y Orientación Familiar (Número 9). https://doi.org/10.36576/summa.28138

Medeiros, S. E., \& Pereyra Bordón, R. (s. f.). Colaboración Sector Defensa Pública en confrontación a las vulnerabilidades de seguridad Hemisféricas . Revista del Colegio Interamericano de Defensa, 2015, 115-131. 
Nd2. (2020). Nd2 Entrevista Experto.

Nd3. (2020). Nd3 Entrevista Experto.

Nm1. (2020). Nm1 Entrevista Experto.

No1. (2020). No1 Entrevista Experto.

NVivo. (s. f.). Web Page Glossary words list. Recuperado 3 de julio de 2020, de http://help-nv8-es. qsrinternational.com/whgdata/whlstg0.htm

Oliva, P. (2009). Construcción de lista de chequeo en salud: Una metodología para su construcción. En Serie Cuadernos de Redes: Ministerio de Salud (Número 24). http://www.bibliotecaminsal.cl/wp/ wp-content/uploads/2016/03/24.pdf

Página Web Centro Nacional de Infraestructuras Críticas CNPIC. (s. f.). http://www.cnpic.es/ Presentacion/index.html

Planeación, D. N. de. (s. f.). Seguridad efectiva y justicia transparente para que todos vivamos con libertad y en democracia. https://www.dnp.gov.co/DNPN/Plan-Nacional-de-Desarrollo/Paginas/ Pilares-del-PND/Legalidad/Seguridad-efectiva-y-justicia-transparente-para-que-todosvivamos-con-libertad-y-en-democracia.aspx

Quintana Peña, A. (2006). Metodología de Investigación Científica Cualitativa. Universidad Nacional Mayor de San Marcos, 47-84.

Sanabria Rangel, P. E. (2016). Investigación en ciencias sociales y de gestión: Guía para el desarrollo de marcos metodológicos y procesos de investigación.

Sanabria Rangel, P. E. (2020). Seminario de Investigación. En Universidad Externado de Colombia (pp. 1-44). http://administracion.uexternado.edu.co/publicaciones/default.asp?codigo=31 
Spreng, D. (2014). Transdisciplinary energy research - Reflecting the context. Energy Research and Social Science, 1, 65-73. https://doi.org/10.1016/j.erss.2014.02.005

Stirling, A. (2014). Transforming power: Social science and the politics of energy choices. Energy Research and Social Science, 1, 83-95. https://doi.org/10.1016/j.erss.2014.02.001

Universidad Alberto Hurtado. (s. f.). El proceso de investigación cualitativa. 